\title{
CUBIC SPLINE COALESCENCE FRACTAL INTERPOLATION THROUGH MOMENTS
}

\author{
A. K. B. CHAND* and G. P. KAPOOR ${ }^{\dagger}$ \\ Department of Mathematics \\ Indian Institute of Technology Kanpur \\ Kanpur-208016, India \\ *akbchand@gmail.com \\ ${ }^{\dagger} g p @ i i t k . a c . i n$
}

Received April 25, 2006

Accepted September 18, 2006

\begin{abstract}
This paper generalizes the classical cubic spline with the construction of the cubic spline coalescence hidden variable fractal interpolation function (CHFIF) through its moments, i.e. its second derivative at the mesh points. The second derivative of a cubic spline CHFIF is a typical fractal function that is self-affine or non-self-affine depending on the parameters of the generalized iterated function system. The convergence results and effects of hidden variables are discussed for cubic spline CHFIFs.
\end{abstract}

Keywords: Iterated Function Systems; Fractal Interpolation Functions; Coalescence; Cubic Spline; Moments; Self-Affine; Non-Self-Affine; Convergence.

\section{INTRODUCTION}

Fractals represent powerful techniques to approximate natural objects such as trees, clouds, landscapes, glaciers, and waves that cannot be described by using classical geometry. With the introduction of the term fractals by Mandelbrot, ${ }^{1}$ the fractal geometry has been successfully used in various domains such as economics, ${ }^{2}$ physics, ${ }^{3}$ graphics, ${ }^{4}$ life sciences,${ }^{5}$ signal processing, ${ }^{6}$ image processing, ${ }^{7}$ etc. Fractal interpolation function (FIF) is

\footnotetext{
* Presently at Departamento de Matemática Aplicada, Centro Politécnico Superior de Ingenieros, Universidad de Zaragoza. C/Mariá de Luna, 3, 50018, Zaragoza, España . Mailing address for offprints: Plot No.: 3424/5090 (Sraddha Nivas), Jharana Sali, Badagada Brit colony, Bhubaneswar, Orissa, India-751018.
} 
introduced by Barnsley ${ }^{8,9}$ through the iterated function system (IFS) to model a large class of self-affine or self-similar objects. The construction of a FIF is based on the fixed point of the ReadBajraktarević operator that preserves self-affinity or self-similarity.

The term "hidden variable" has been introduced by Barnsley et al. ${ }^{10}$ and Massopust. ${ }^{11}$ The hidden variable FIF (HFIF) is more diverse, appealing and irregular than FIF for the same set of interpolation data since the values of hidden variable FIF continuously depend on all parameters which define it. Since the HFIF is the projection of a vector valued function, it is usually non-self-affine in nature. However, in practical applications of FIFs, the interpolation data might be generated simultaneously from self-affine or non-self-affine functions. ${ }^{12}$ To approximate self-affine or non-self-affine functions simultaneously, the coalescence hidden variable FIF (CHFIF) is introduced (see for instance Refs. 13 and 14).

The existence of a differentiable FIF or spline FIF (SFIF) is introduced by Barnsley and Harrington. ${ }^{15}$ However, the construction of SFIFs with only a fixed type of boundary conditions is allowed in their construction. The construction of SFIFs with any type of boundary conditions is given in Refs. 14, 16 to 18. The derivative of a SFIF is a typical fractal that is self-affine in nature. CHFIFs can be integrated successively in order to get more diverse and appealing spline CHFIFs, where hidden variables play significant role in their shapes. Such type of splines are useful in practical applications since their derivatives can be either of self-affine or nonself-affine fractal functions. The construction and convergence analysis of cubic spline CHFIFs have practical importance in view of significant applications of cubic splines in science and engineering problems. ${ }^{19,20}$

In the present paper, the construction of cubic spline CHFIF $f_{1}(x)$ on a mesh $\Delta$ is developed through moments $M_{n}^{*}=f_{1}{ }^{\prime \prime}\left(x_{n}\right), n=0,1,2, \ldots, N$, with any type of boundary conditions as in the classical cubic spline. The advantage of such a construction is that, for a prescribed data and boundary conditions, one can have infinite number of cubic spline CHFIFs that are self-affine or nonself-affine, depending on choice of hidden variables and boundary conditions of the associated cubic spline fractal functions. The convergence results of cubic spline CHFIFs on two classes of sequence of uniform or non-uniform meshes are proved for the data generating function $\Phi(x)$, where $\Phi \in$ $C^{r}\left[x_{0}, x_{N}\right]$, for $r=2,3$, or 4 .

The organization of this paper is as follows. In Sec. 2, we discuss the construction of cubic spline CHFIFs through its moments. Our construction admits all types of boundary conditions as in classical cubic splines. The convergence results of cubic spline CHFIFs are described on two classes of sequence of meshes in Sec. 3. Finally, effects of hidden variables on the cubic spline CHFIFs are illustrated through suitably chosen examples in Sec. 4.

\section{CUBIC SPLINE HFIF}

First, we discuss in Sec. 2.1 the basics of CHFIFs. The construction of cubic spline CHFIFs is described in Sec. 2.2.

\subsection{Basics of CHFIFs}

Let $x_{0}<x_{1}<\cdots<x_{N}$ be a partition of an interval $I=\left[x_{0}, x_{N}\right] \subset \mathbb{R}$ and $\left\{\left(x_{n}, y_{n}\right) \in I \times \mathbb{R}: n=0,1,2\right.$, $\ldots, N\}$ be a set of data points. This data set is extended to a generalized set of data $\left\{\left(x_{n}, y_{n}, z_{n}\right) \in\right.$ $\left.\mathbb{R}^{3}: n=0,1,2, \ldots, N\right\}$ with real parameters $z_{n}, n$ $=0,1,2, \ldots, N$. Set, $\tilde{g}_{1}=\operatorname{Min}_{n} y_{n}, \tilde{g}_{2}=\operatorname{Max}_{n} y_{n}$, $\tilde{h}_{1}=\operatorname{Min}_{n} z_{n}, \tilde{h}_{2}=\operatorname{Max}_{\mathrm{n}} z_{n}$, and $K=I \times D$, where $D=J_{1} \times J_{2}, J_{1}$ and $J_{2}$ are suitable compact sets in $\mathbb{R}$ such that $\left[\tilde{g}_{1}, \tilde{g}_{2}\right] \subseteq J_{1},\left[\tilde{h}_{1}, \tilde{h}_{2}\right] \subseteq J_{2}$. Let $L_{n}: I \rightarrow I_{n}=\left[x_{n-1}, x_{n}\right]$ be a contraction map satisfying

$$
L_{n}\left(x_{0}\right)=x_{n-1}, \quad L_{n}\left(x_{N}\right)=x_{n} .
$$

Let $F_{n}: K \rightarrow D$ be a vector valued function satisfying

$$
\left.\begin{array}{l}
F_{n}\left(x_{0}, y_{0}, z_{0}\right)=\left(y_{n-1}, z_{n-1}\right), \\
F_{n}\left(x_{N}, y_{N}, z_{N}\right)=\left(y_{n}, z_{n}\right), \\
d\left(F_{n}(x, y, z), F_{n}\left(x^{*}, y^{*}, z^{*}\right)\right) \\
\quad \leq s d_{E}\left((y, z),\left(y^{*}, z^{*}\right)\right),
\end{array}\right\}
$$

for $n=1,2, \ldots, N$, where $(x, y, z),\left(x^{*}, y^{*}, z^{*}\right) \in K$, $0 \leq s<1, d$ is the sup. metric on $K$, and $d_{E}$ is the Euclidean metric on $\mathbb{R}^{2}$. In order to define the CHFIF, functions $L_{n}$ and $F_{n}$ are chosen such that $L_{n}(x)=a_{n} x+b_{n}$ and

$$
\begin{aligned}
F_{n}(x, y, z) & =A_{n}(y, z)^{T}+\left(p_{n}(x), q_{n}(x)\right)^{T} \\
& =\left(F_{n}^{1}(x, y, z), F_{n}^{2}(x, z)\right)^{T}
\end{aligned}
$$

where $A_{n}$ is an upper triangular matrix $\left(\begin{array}{cc}\alpha_{n} & \beta_{n} \\ 0 & \gamma_{n}\end{array}\right)$ and $p_{n}(x), q_{n}(x)$ are continuous functions having two free parameters. These parameters can be 
determined by using Eq. (2.2). We choose $\alpha_{n}$ as free variable with $\left|\alpha_{n}\right|<1$ and $\beta_{n}$ as constrained free variable with respect to $\gamma_{n}$ such that $\left|\beta_{n}\right|+\left|\gamma_{n}\right|<1$. The generalized IFS that is needed for construction of a CHFIF corresponding to the data $\left\{\left(x_{n}, y_{n}, z_{n}\right)\right.$ : $n=0,1, \ldots, N\}$ is now defined as

$$
\begin{array}{r}
\left\{\mathbb{R}^{3} ; \omega_{n}(x, y, z)=\left(L_{n}(x), F_{n}(x, y, z)\right),\right. \\
n=1,2, \ldots, N\} .
\end{array}
$$

It is known ${ }^{10}$ that the IFS defined in Eq. (2.4) associated with the data $\left\{\left(x_{n}, y_{n}, z_{n}\right): n=0,1, \ldots, N\right\}$ is hyperbolic with respect to a metric $\tau$ that is equivalent to the Euclidean metric on $\mathbb{R}^{3}$. Hence, there exists a unique non-empty compact set $G \subseteq$ $\mathbb{R}^{3}$, called as attractor of the IFS (2.4), such that $G=\bigcup_{i=1}^{N} \omega_{i}(G)$. This attractor $G$ provides the existence of a unique vector valued interpolant $f$ in the following proposition.

Proposition 2.1. ${ }^{10}$ The attractor $G$ of the IFS defined by Eq. (2.4) is the graph of the continuous vector valued function $f: I \rightarrow D$ such that $f\left(x_{n}\right)=$ $\left(y_{n}, z_{n}\right)$ for all $n=1,2, \ldots, N$, i.e., $G=\{(x, y, z)$ : $x \in I$ and $f(x)=(y(x), z(x))\}$.

Proposition 2.1 gives that the graph of the vector valued function $f(x)=\left(f_{1}(x), f_{2}(x)\right)$ is the attractor of the IFS $\left\{\mathbb{R}^{3} ; \omega_{n}(x, y, z), n=1,2, \ldots, N\right\}$ if and only if the fixed point $f$ of Read-Bajraktarević operator $T$ on the space of continuous vector valued functions from $I$ to $D$ satisfies

$$
\begin{aligned}
T f(x)=f(x)= & F_{n}\left(L_{n}^{-1}(x), f\left(L_{n}^{-1}(x)\right)\right), \\
& x \in I_{n}, \quad n=1,2, \ldots, N .
\end{aligned}
$$

The image $T f$ of the vector valued function $f$ can be written component wise as $\left(T_{1} f_{1}, T_{2} f_{2}\right)$, where $T_{1}$ and $T_{2}$ are component wise Read-Bajraktarević operators from $I$ to $\mathbb{R}$. The function $f_{1}(x)$ in the projection $\left\{\left(x, f_{1}(x)\right): x \in I\right\}$ of the attractor $G$ on $\mathbb{R}^{2}$, is called coalescence FIF or coalescence hidden variable FIF (CHFIF) for the given data $\left\{\left(x_{n}, y_{n}\right): n=0,1, \ldots, N\right\}$. It is easily seen that CHFIFs satisfy the following functional equation for $x \in I$.

$$
\begin{aligned}
T_{1} f_{1}\left(L_{n}(x)\right) & =f_{1}\left(L_{n}(x)\right) \\
& =F_{n}^{1}\left(x, f_{1}(x), f_{2}(x)\right) \\
& =\alpha_{n} f_{1}(x)+\beta_{n} f_{2}(x)+p_{n}(x) .
\end{aligned}
$$

Similarly, the function $f_{2}(x)$ in the projection $\left\{\left(x, f_{2}(x)\right): x \in I\right\}$ of the attractor $G$ is a selfaffine fractal function that interpolates the data $\left\{\left(x_{n}, z_{n}\right): n=0,1, \ldots, N\right\}$ and satisfies the following functional equation.

$$
\begin{aligned}
T_{2} f_{2}\left(L_{n}(x)\right) & =f_{2}\left(L_{n}(x)\right) \\
& =F_{n}^{2}\left(x, f_{2}(x)\right) \\
& =\gamma_{n} f_{2}(x)+q_{n}(x), \quad x \in I .
\end{aligned}
$$

Since the projection of the attractor is not always union of affine transformations of itself, HFIFs are generally non-self-affine by nature. By choosing $y_{n}=z_{n}$ and $\alpha_{n}+\beta_{n}=\gamma_{n}$, CHFIF $f_{1}(x)$ obtained as the projection on $\mathbb{R}^{2}$ of the attractor of the IFS (2.4) coincides with a self-affine fractal function $f_{2}(x)$ for the same interpolation data. Hence, the CHFIF is self-affine or self-similar in this case. This type of CHFIFs can be used to approximate the random steps of Gaussian, increments of the fractional Brownian function and wave-height functions. ${ }^{3}$

\subsection{Construction of Cubic Spline CHFIFs}

A function $S(x)$ is said to be a cubic spline on a grid $x_{0}<x_{1}<\cdots<x_{N}$ if it satisfies (i) $S(x)$ is a polynomial of degree 3 on each subinterval $\left[x_{n-1}, x_{n}\right]$ and (ii) $S^{r}(x)$ is continuous on $\left[x_{0}, x_{N}\right]$ for $r=0,1,2$. The following proposition provides the existence of a SFIF.

Proposition 2.2. ${ }^{16}$ Let $\left\{\left(x_{n}, y_{n}\right): n=0,1,2, \ldots, N\right\}$ be the interpolation data with $x_{0}<x_{1}<x_{2}<\ldots<$ $x_{N}$. Let $L_{n}(x)=a_{n} x+b_{n}$ that satisfies Eq. (2.1) and $F_{n}(x, y)=\alpha_{n} y+q_{n}(x)$ for $n=1,2, \ldots, N$. Suppose for some integer $r \geq 0,\left|\alpha_{n}\right|<a_{n}^{r}$, and $q_{n} \in C^{r}\left[x_{0}, x_{N}\right] ; n=1,2, \ldots, N$. Let

$$
\begin{aligned}
F_{n, k}(x, y) & =\frac{\alpha_{n} y+q_{n}^{(k)}}{a_{n}^{k}}, \\
x_{0, k} & =\frac{q_{1}^{(k)}\left(x_{0}\right)}{a_{1}^{k}-\alpha_{1}}, \\
x_{N, k} & =\frac{q_{N}^{(k)}\left(x_{N}\right)}{a_{N}^{k}-\alpha_{N}}, \quad k=1,2, \ldots, r .
\end{aligned}
$$

If $F_{n-1, k}(x, y)\left(x_{N}, y_{N, k}\right)=F_{n, k}\left(x_{0}, y_{o, k}\right)$ for $n=$ $2,3, \ldots, N$ and $k=1,2, \ldots, r$, then $\left\{\left(L_{n}(x)\right.\right.$, $\left.\left.F_{n}(x, y)\right)\right\}_{n=1}^{N}$ determines a FIF $f \in C^{r}\left[x_{0}, x_{N}\right]$ and $f^{(k)}, k=1,2, \ldots, r$, is the FIF determined by $\left\{\left(L_{n}(x), F_{n, k}(x, y)\right)\right\}_{n=1}^{N}$.

Based on the Proposition 2.2, we define the cubic spline CHFIF $f_{1}$ through moments, $M_{n}^{*}=f_{1}^{\prime \prime}\left(x_{n}\right)$, $n=0,1,2, \ldots, N$, as follows. 
Definition 2.1. A function $f_{1}(x)$ (or $f_{1}(Y ; x)$ ) is called a cubic spline CHFIF interpolating to the data set $\left\{\left(x_{n}, y_{n}\right): n=0,1, \ldots, N\right\}$ with mesh $\Delta: x_{0}<x_{1}<x_{2}<\cdots<x_{N}$, if (i) $f_{1} \in$ $C^{2}\left[x_{0}, x_{N}\right]$, (ii) $f_{1}$ satisfies the interpolation conditions $f_{1}\left(x_{n}\right)=y_{n}, n=0,1, \ldots, N$, and (iii) the graph of $f_{1}$ is the projection of the attractor of a IFS, $\left\{\mathbb{R}^{3} ; \omega_{n}(x, y, z), n=1,2, \ldots, N\right\}$ to $\mathbb{R}^{2}$, where for $n=1,2, \ldots, N, \omega_{n}(x, y)=$ $\left(L_{n}(x), F_{n}(x, y, z)\right), \quad L_{n}(x)$ is defined as in Eq. (2.1), $\quad F_{n}(x, y, z)=\left(a_{n}^{2}\left(\alpha_{n} y+\beta_{n} z+p_{n}(x)\right)\right.$, $\left.a_{n}^{2}\left(\gamma_{n} z+q_{n}(x)\right)\right), 0<\left|\alpha_{n}\right|<1,0<\left|\beta_{n}\right|+\left|\gamma_{n}\right|<1$, and $p_{n}(x), q_{n}(x)$ are suitable cubic polynomials.
Denote moments of self-affine fractal function as $M_{n}=f_{2}{ }^{\prime \prime}\left(x_{n}\right)$ for $n=0,1,2, \ldots, N$. The moments $M_{n}^{*}$ and $M_{n} ; n=0,1,2, \ldots, N$, are used to determine the polynomials $p_{n}(x)$ and $q_{n}(x)$ (see Appendix). Thus, the desired IFS for the construction of the cubic spline CHFIF $f_{1}$ is given by

$$
\begin{array}{r}
\left\{\mathbb{R}^{3} ; \omega_{n}(x, y, z)=\left(L_{n}(x), F_{n}(x, y, z)\right.\right. \\
\left.=\left(F_{n}^{1}(x, y, z), F_{n}^{2}(x, z)\right)\right), \\
n=1,2, \ldots, N\},
\end{array}
$$

where $L_{n}(x)=a_{n} x+b_{n}$,

$$
\begin{aligned}
& F_{n}^{1}(x, y, z)=a_{n}^{2}\left\{\alpha_{n} y+\beta_{n} z+\frac{\left(M_{n}^{*}-\alpha_{n} M_{N}^{*}-\beta_{n} M_{N}\right)\left(x-x_{0}\right)^{3}}{6\left(x_{N}-x_{0}\right)}+\left(M_{n-1}^{*}-\alpha_{n} M_{0}^{*}-\beta_{n} M_{0}\right)\right. \\
& \quad \times \frac{\left(x_{N}-x\right)^{3}}{6\left(x_{N}-x_{0}\right)}-\frac{\left(M_{n-1}^{*}-\alpha_{n} M_{0}^{*}-\beta_{n} M_{0}\right)\left(x_{N}-x_{0}\right)\left(x_{N}-x\right)}{6}-\left(M_{n}^{*}-\alpha_{n} M_{N}^{*}-\beta_{n} M_{N}\right) \\
& \left.\quad \times \frac{\left(x_{N}-x_{0}\right)\left(x-x_{0}\right)}{6}+\left(\frac{y_{n-1}}{a_{n}^{2}}-\alpha_{n} y_{0}-\beta_{n} z_{0}\right) \frac{x_{N}-x}{x_{N}-x_{0}}+\left(\frac{y_{n}}{a_{n}^{2}}-\alpha_{n} y_{N}-\beta_{n} z_{N}\right) \frac{x-x_{0}}{x_{N}-x_{0}}\right\},
\end{aligned}
$$

and

$$
\begin{aligned}
F_{n}^{2}(x, z)= & a_{n}^{2}\left\{\gamma_{n} z+\frac{\left(M_{n}-\gamma_{n} M_{N}\right)\left(x-x_{0}\right)^{3}}{6\left(x_{N}-x_{0}\right)}+\frac{\left(M_{n-1}-\gamma_{n} M_{0}\right)\left(x_{N}-x\right)^{3}}{6\left(x_{N}-x_{0}\right)}\right. \\
& -\frac{\left(M_{n-1}-\gamma_{n} M_{0}\right)\left(x_{N}-x_{0}\right)\left(x_{N}-x\right)}{6}-\frac{\left(M_{n}-\gamma_{n} M_{N}\right)\left(x_{N}-x_{0}\right)\left(x-x_{0}\right)}{6} \\
& \left.+\left(\frac{z_{n-1}}{a_{n}^{2}}-\gamma_{n} z_{0}\right) \frac{x_{N}-x}{x_{N}-x_{0}}+\left(\frac{z_{n}}{a_{n}^{2}}-\gamma_{n} z_{N}\right) \frac{x-x_{0}}{x_{N}-x_{0}}\right\}
\end{aligned}
$$

The projection of the attractor $G$ of the IFS given by Eq. (2.8), i.e. $\left\{\left(x, f_{1}(x)\right) \mid x \in I\right\}$ is the graph of the required cubic spline CHFIF that may be self-affine or non-self-affine depending on the hidden variables. Suppose that the data $\left\{\left(x_{n}, y_{n}\right): n=\right.$ $0,1,2, \ldots, N\}$ is generated by a continuous function $\Phi$ that is approximated by the cubic spline CHFIF $f_{1}$. Then $f_{1}$ is called (i) the complete cubic spline CHFIF if it has boundary conditions of TypeI, i.e. $f_{1}^{\prime}\left(x_{0}\right)=\Phi^{\prime}\left(x_{0}\right), f_{1}^{\prime}\left(x_{N}\right)=\Phi^{\prime}\left(x_{N}\right)$; (ii) the natural cubic spline CHFIF with $M_{0}^{*}=M_{N}^{*}=$ 0 if it has boundary conditions of Type-II, i.e. $f_{1}^{\prime \prime}\left(x_{0}\right)=\Phi^{\prime \prime}\left(x_{0}\right)=M_{0}^{*}, \quad f_{1}^{\prime \prime}\left(x_{N}\right)=\Phi^{\prime \prime}\left(x_{N}\right)=$ $M_{N}^{*}$; and (iii) the periodic cubic spline CHFIF if it has boundary conditions of Type-III, i.e. $f_{1}\left(x_{0}\right)=$ $f_{1}\left(x_{N}\right), f_{1}^{\prime}\left(x_{0}\right)=f_{1}^{\prime}\left(x_{N}\right), f_{1}^{\prime \prime}\left(x_{0}\right)=f_{1}^{\prime \prime}\left(x_{N}\right)$.

Remark 2.1. (1) If free variables $\alpha_{n}=0$ and constrained free variables $\beta_{n}=0 ; n=1,2, \ldots, N$, $F_{n}^{1}(x, y, z)$ reduces to a cubic polynomial in each sub-interval of $I$. Hence, the IFS (2.8) generates the classical cubic spline $S(x)$ as a special case of the cubic spline CHFIF.

(2) In general, a cubic spline CHFIF is not selfaffine as it is the projection of attractor of a nondiagonal IFS. But, if $y_{n}=z_{n}, n=0,1,2, \ldots, N$, $\alpha_{n}+\beta_{n}=\gamma_{n}$ for $n=1,2, \ldots, N$, and $f_{1}, f_{2}$ have the same boundary conditions, the cubic spline CHFIF is self-affine in nature, i.e. second derivative of the cubic spline CHFIF is a typical self-affine fractal function.

(3) If $f_{1}$ is periodic, the necessary condition for the existence of the periodic cubic spline CHFIF for prescribed moments $M_{n}$ is given by

$$
\begin{gathered}
\sum_{n=1}^{N}\left[\left(h_{n}+h_{n+1}\right) M_{n}^{*}-2 \alpha_{n} h_{n} M_{N}^{*}-2 a_{n} \beta_{n}\left(f_{2}{ }^{\prime}\left(x_{N}\right)\right.\right. \\
\left.\left.\quad-f_{2}{ }^{\prime}\left(x_{0}\right)\right)-\beta_{n} h_{n}\left(M_{0}+M_{N}\right)\right]=0 .
\end{gathered}
$$

With $\alpha_{n}=0$ and $\beta_{n}=0$ for $n=1,2, \ldots, N$, Eq. (2.11) reduces to the necessary condition for 
the existence of the periodic classical cubic spline associated with $M_{n} \cdot{ }^{21}$ Also, for $\beta_{n}=0$ for $n=$ $1,2, \ldots, N$, Eq. (2.11) reduces to the necessary condition for the existence of the periodic SFIF associated with $M_{n}^{*} \cdot{ }^{16}$

\section{CONVERGENCE OF CUBIC SPLINE CHFIFS}

Let $\mathcal{G}^{*}=\left\{f \in C^{2}\left(I, \mathbb{R}^{2}\right): f\left(x_{n}\right)=\left(y_{n}, z_{n}\right), n=\right.$ $0,1,2, \ldots, N\}$, where $I=\left[x_{0}, x_{N}\right]$. Let $f_{1}$ and $f_{2}$ be the components of the vector valued function $f$ such that $f=\left(f_{1}, f_{2}\right)$. From Eqs. (2.6) and (2.9), the cubic spline CHFIF satisfies the implicit relation

$$
\begin{array}{r}
f_{1}\left(L_{n}(x)\right)=a_{n}^{2}\left(\alpha_{n} f_{1}(x)+\beta_{n} f_{2}(x)+p_{n}(x)\right), \\
x \in I
\end{array}
$$

and from Eqs. (2.7) and (2.10), the self-affine cubic spline fractal function satisfies the functional relation

$$
f_{2}\left(L_{n}(x)\right)=a_{n}^{2}\left(\gamma_{n} f_{2}(x)+q_{n}(x)\right), x \in I,
$$

where $p_{n}(x)$ and $q_{n}(x)$ are cubic polynomials for $n=1,2, \ldots, N$. In this section, we assume that for $n=1,2, \ldots, N ;\left|\beta_{n}\right|+\left|\gamma_{n}\right| \leq s<1$ and $\left|\alpha_{n}\right| \leq s^{*}<$ 1 , where $s$ and $s^{*}$ are some fixed real numbers. In view of Eqs. (2.9) and (2.10), denote $p_{n}\left(\alpha_{n}, \beta_{n}, x\right) \equiv$ $p_{n}(x)$ and $q_{n}\left(\gamma_{n}, x\right) \equiv q_{n}(x)$ for $n=1,2, \ldots, N$. Further, let for $x \in I_{n}, n=1,2, \ldots, N, q_{n}(x)$ satisfies

$$
\left|\frac{\partial^{1+r} q_{n}\left(\tau_{n}, x\right)}{\partial \gamma_{n} \partial x^{r}}\right| \leq K_{r}
$$

where $\left|\tau_{n}\right| \in\left(0, s a_{n}^{r}\right)$ and $K_{r}$ is a positive constant. We need the following lemma to prove our main convergence Theorems 3.1 to 3.3 .

Lemma 3.1. Let $f_{1}(x)$ be the cubic spline CHFIF through generalized interpolation data and $S(x)$ be the classical cubic spline with respect to the mesh $\Delta: x_{0}<x_{1}<\ldots<x_{N}$, interpolating $\left\{y_{0}, y_{1}, \ldots\right.$, $\left.y_{N}\right\}$ at the mesh points with same type of boundary conditions. Suppose, there exist positive constants $K_{r}^{*}, K_{r}^{* *}, r=0,1,2$ such that

$$
\begin{aligned}
& \left|\frac{\partial^{1+r} p_{n}\left(\xi_{n}, \beta_{n}, x\right)}{\partial \alpha_{n} \partial x^{r}}\right| \leq K_{r}^{*}, \\
& \left|\frac{\partial^{1+r} p_{n}\left(\alpha_{n}, \eta_{n}, x\right)}{\partial \beta_{n} \partial x^{r}}\right| \leq K_{r}^{* *}
\end{aligned}
$$

for $\left|\xi_{n}\right| \in\left(0, s^{*} a_{n}^{r}\right), \quad\left|\eta_{n}\right| \in\left(0, s a_{n}^{r}\right), x \in I_{n}, \quad n=$ $1,2, \ldots, N$, and $r=0,1,2$. Then,

$$
\begin{aligned}
& \left\|f_{1}^{(r)}-S^{(r)}\right\|_{\infty} \\
& \max _{1 \leq n \leq N}\left|\alpha_{n}\right|\left(\left\|S^{(r)}\right\|_{\infty}+K_{r}^{*}\right) \\
& \leq\|\Delta\|^{2-r} \frac{+\max _{1 \leq n \leq N}\left|\beta_{n}\right|\left(\left\|f_{2}{ }^{(r)}\right\|_{\infty}+K_{r}^{* *}\right)}{|I|^{2-r}-\|\Delta\|^{2-r} \max _{1 \leq n \leq N}\left|\alpha_{n}\right|}, \\
& r=0,1,2,
\end{aligned}
$$

where $|I|$ is the length of the interval $I$.

Proof. Denote $\mathcal{B}_{r}^{*}=\left(\bigotimes_{n=1}^{N}\left[-s^{*} a_{n}^{r}, s^{*} a_{n}^{r}\right] ; \bigotimes_{n=1}^{N}\right.$ $\left.\left(-s a_{n}^{r}, s a_{n}^{r}\right)\right)$ for $r=0,1,2$. Since the proof is analogous for any value of $r$, we prove it for $r=0$ as follows. Let $(\alpha, \beta)=\left(\alpha_{1}, \alpha_{2}, \ldots, \alpha_{N} ; \beta_{1}, \beta_{2}, \ldots, \beta_{N}\right) \in$ $\mathcal{B}_{0}^{*}$. For a given self-affine cubic spline fractal function $f_{2}$ and given boundary conditions, cubic spline CHFIF $f_{1}$ is unique for an element $(\alpha, \beta)$ in $\mathcal{B}_{0}^{*}$. So, using Eq. (3.1), the component wise ReadBajraktarević operator $T_{1}^{*}{ }_{\left(\alpha^{*}, \beta^{*}\right)}$ of $T^{*}: \mathcal{G}^{*} \rightarrow \mathcal{G}^{*}$, can be written as for $x \in I_{n}$ and $n=1,2, \ldots, N$,

$$
\begin{aligned}
T_{1(\alpha, \beta)}^{*} f_{1}^{*}(x)= & a_{n}^{2}\left[\alpha_{n} f_{1}^{*}\left(L_{n}^{-1}(x)\right)\right. \\
& +\beta_{n} f_{2}{ }^{*}\left(L_{n}^{-1}(x)\right) \\
& \left.+p_{n}\left(\alpha_{n}, \beta_{n}, L_{n}^{-1}(x)\right)\right] .
\end{aligned}
$$

Suppose $f_{1}$ is the fixed point of $T_{1}^{*}(\alpha, \beta)$, where $(\alpha ; \beta) \in \mathcal{B}_{0}^{*}$ such that $\alpha_{n} \neq 0$ and $\beta_{m} \neq 0$ for some $n$ and $m$. Also, if $\left(\alpha^{*}, \beta^{*}\right)=(0,0, \ldots, 0 ; 0,0, \ldots, 0) \in$ $\mathcal{B}_{0}^{*}$, then the classical cubic spline $S$ is the fixed point of $T_{1}^{*}{ }_{\left(\alpha^{*}, \beta^{*}\right)}$ with the prescribed boundary conditions. Hence, by Eq. (3.6), for $x \in I_{n}, n=$ $1,2, \ldots, N$,

$$
\begin{aligned}
\mid T_{1(\alpha, \beta)}^{*} & f_{1}(x)-T_{1(\alpha, \beta)}^{*} S(x) \mid \\
= & \mid a_{n}^{2}\left[\alpha_{n} f_{1}\left(L_{n}^{-1}(x)\right)+\beta_{n} f_{2}\left(L_{n}^{-1}(x)\right)\right. \\
& \left.+p_{n}\left(\alpha_{n}, \beta_{n}, L_{n}^{-1}(x)\right)\right] \\
& -a_{n}^{2}\left[\alpha_{n} S\left(L_{n}^{-1}(x)\right)+\beta_{n} f_{2}\left(L_{n}^{-1}(x)\right.\right. \\
& \left.+p_{n}\left(\alpha_{n}, \beta_{n}, L_{n}^{-1}(x)\right)\right] \mid \\
= & a_{n}^{2}\left|\alpha_{n}\right|\left|f_{1}\left(L_{n}^{-1}(x)\right)-S\left(L_{n}^{-1}(x)\right)\right| \\
\leq & \frac{\|\Delta\|^{2}}{|I|^{2}} \max _{1 \leq n \leq N}\left|\alpha_{n}\right|\left\|f_{1}-S\right\|_{\infty} .
\end{aligned}
$$

The above inequality gives

$$
\begin{aligned}
& \left\|T_{1(\alpha, \beta)}^{*} f_{1}-T_{1(\alpha, \beta)}^{*} S\right\|_{\infty} \\
& \quad \leq \frac{\|\Delta\|^{2}}{|I|^{2}} \max _{1 \leq n \leq N}\left|\alpha_{n}\right|\left\|f_{1}-S\right\|_{\infty} .
\end{aligned}
$$


Now, using the mean value theorem and Eq. (3.5), for $x \in I_{n}, n=1,2, \cdots, N$,

$$
\begin{aligned}
& \mid T_{1}^{*}(\alpha, \beta) \\
& \quad=\left|a_{n}^{2}\left[\alpha_{n} S\left(L_{n}^{-1}(x)\right)+\beta_{n} f_{2}\left(L_{n}^{-1}(x)\right)+p_{n}\left(\alpha_{n}, \beta_{n}, L_{n}^{-1}(x)\right)-p_{n}\left(0,0, L_{n}^{-1}(x)\right)\right]\right| \\
& \quad \leq a_{n}^{2}\left\{\left|\alpha_{n}\right|\|S\|_{\infty}+\left|\beta_{n}\right|\left\|f_{2}\right\|_{\infty}+\left|\alpha_{n}\right|\left|\frac{\partial p_{n}\left(\xi_{n}, \beta_{n}, L_{n}^{-1}(x)\right)}{\partial \alpha_{n}}\right|+\left|\beta_{n}\right|\left|\frac{\partial p_{n}\left(0, \eta_{n}, L_{n}^{-1}(x)\right)}{\partial \beta_{n}}\right|\right\} \\
& \quad \leq \frac{\|\Delta\|^{2}}{|I|^{2}}\left\{\max _{1 \leq n \leq N}\left|\alpha_{n}\right|\left(\|S\|_{\infty}+K_{0}^{*}\right)+\max _{1 \leq n \leq N}\left|\beta_{n}\right|\left(\left\|f_{2}\right\|_{\infty}+K_{0}^{* *}\right)\right\} .
\end{aligned}
$$

It follows that

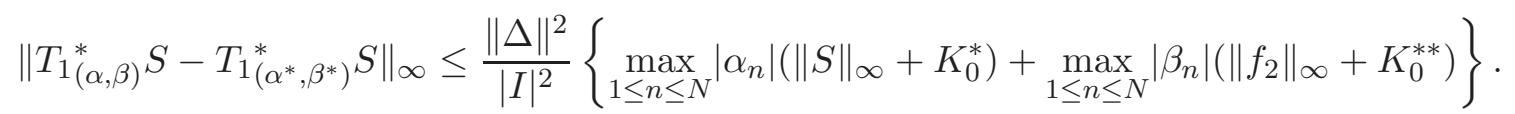

Using inequalities (3.7) and (3.8) in

$$
\begin{aligned}
\left\|f_{1}-S\right\|_{\infty} & =\left\|T_{1}^{*}{ }_{(\alpha, \beta)}^{*} f_{1}-T_{1_{\left(\alpha^{*}, \beta^{*}\right)}^{*}}^{*} S\right\|_{\infty} \\
& \leq\left\|T_{1_{(\alpha, \beta)}^{*}}^{*} f_{1}-T_{1_{(\alpha, \beta)}^{*}}^{*} S\right\|_{\infty}+\left\|T_{1}^{(\alpha, \beta)}{ }^{*} S-T_{1_{\left(\alpha^{*}, \beta^{*}\right)}^{*}}^{*}\right\|_{\infty}
\end{aligned}
$$

resulted into

$$
\left\|f_{1}-S\right\|_{\infty} \leq\|\Delta\|^{2} \frac{\max _{1 \leq n \leq N}\left|\alpha_{n}\right|\left(\|S\|_{\infty}+K_{0}^{*}\right)+\max _{1 \leq n \leq N}\left|\beta_{n}\right|\left(\left\|f_{2}\right\|_{\infty}+K_{0}^{* *}\right)}{|I|^{2}-\|\Delta\|^{2} \max _{1 \leq n \leq N}\left|\alpha_{n}\right|}
$$

Hence, Lemma 3.1 is proved for $r=0$. The proof is similar to $r=1,2$, and thus omitted.

Remark 3.1. By assuming $\beta_{n}=0$ and replacing $\alpha_{n}$ by $\gamma_{n}$ and inequality (3.4) by inequality (3.3) in Lemma 3.1, the self-affine cubic spline fractal function $f_{2}$ satisfies the following estimate:

$$
\begin{aligned}
\| f_{2}{ }^{(r)} & -S^{(r)} \|_{\infty} \\
& \leq \frac{\|\Delta\|^{2-r} s}{|I|^{2-r}-\|\Delta\|^{2-r} s}\left(\left\|S^{(r)}\right\|_{\infty}+K_{r}\right),
\end{aligned}
$$

when $f_{2}$ and $S$ are constructed with the same boundary conditions.

Let $\Delta_{k}$ be a sequences of meshes on $\left[x_{0}, x_{N}\right]$ as

$$
\Delta_{k}: x_{0}=x_{k, 0}<x_{k, 1}<\cdots<x_{k, N_{k}}=x_{N} .
$$

Denote, $h_{k, n_{k}}=x_{k, n_{k}}-x_{k, n_{k}-1}$ and $\left\|\Delta_{k}\right\|=$ $\max _{1 \leq n_{k} \leq N_{k}} h_{k, n_{k}}$. We prove that the sequence of cubic spline CHFIFs $\left\{f_{1 \Delta_{k}}(x)\right\}$ with boundary conditions of Type-I, Type-II, or Type-III converge to the data generating function $\Phi(x)$ on a sequences of meshes $\left\{\Delta_{k}\right\}$ at the rate of $\left\|\Delta_{k}\right\|^{2}$, when the corresponding self-affine cubic spline fractal function $f_{2 \Delta_{k}}(x)$ is constructed with any one of the boundary conditions of Type-I, Type-II, or TypeIII, where $\Phi \in C^{r}(I), r=2,3$, or 4 . In view of Lemma 3.1, we define two types of sequences of meshes $\left\{\Delta_{k}\right\}$ on $\left[x_{0}, x_{N}\right]$ for study of the convergence of cubic spline CHFIFs to a data generating function, depending upon free variables $\alpha_{k, n_{k}}$ and constrained free variables $\beta_{k, n_{k}}$.

Class A. $\left\{\left\{\Delta_{k}\right\}: \max _{1 \leq n_{k} \leq N_{k}}\left\{\left|\alpha_{k, n_{k}}\right|,\left|\beta_{k, n_{k}}\right|\right\} \leq\left\|\Delta_{k}\right\|<1\right.$ for each $\left.k\right\}$.

Class B. $\left\{\left\{\Delta_{k}\right\}:\left|\alpha_{k, i}\right|>\left\|\Delta_{k}\right\|\right.$ or $\left|\beta_{k, j}\right|>\left\|\Delta_{k}\right\|$ for some $i, j, 1 \leq i, j \leq N_{k}$ and for each $\left.k\right\}$.

For the function $\Phi$ in $C^{2}\left[x_{0}, x_{N}\right]$ generating the interpolation data, the convergence result of cubic spline CHFIFs are given in the following theorem if cubic spline fractal functions $f_{2 \Delta_{k}}$ satisfy any boundary conditions of Type-I, Type-II, or TypeIII on a sequence of meshes.

Theorem 3.1. Let $\Phi \in C^{2}\left[x_{0}, x_{N}\right]$ and cubic spline CHFIFs $f_{1 \Delta_{k}}(x)$ satisfy any boundary conditions of
Type-I, Type-II or Type-III on a sequence of meshes $\left\{\Delta_{k}\right\}$ on $\left[x_{0}, x_{N}\right]$ with $\lim _{k \rightarrow \infty}\left\|\Delta_{k}\right\|=0$. If $\left\{\Delta_{k}\right\}$ is in Class $A$, then

$\left\|\Phi^{(r)}-f_{1} \Delta_{k}^{(r)}\right\|_{\infty}=\circ\left(\left\|\Delta_{k}\right\|^{2-r}\right), \quad r=0,1,2$,

and if $\left\{\Delta_{k}\right\}$ is in Class $B$, then

$\left\|\Phi^{(r)}-f_{1 \Delta_{k}}^{(r)}\right\|_{\infty}=\bigcirc\left(\left\|\Delta_{k}\right\|^{2-r}\right), \quad r=0,1,2$. 
Proof. By Lemma 3.1, with the same boundary conditions, $f_{1 \Delta_{k}}$ and $S_{\Delta_{k}}$ satisfies the following relation on each element of the sequence $\left\{\Delta_{k}\right\}$ for $r=0,1,2$.

$$
\left\|f_{1}^{(r)}-S_{\Delta_{k}}^{(r)}\right\|_{\infty} \leq\left\|\Delta_{k}\right\|^{2-r} \frac{\max _{1 \leq n_{k} \leq N_{k}}\left|\alpha_{k, n_{k}}\right|\left(\left\|S_{\Delta_{k}}^{(r)}\right\|_{\infty}+K_{r}^{*}\right)+\max _{1 \leq n_{k} \leq N_{k}}\left|\beta_{k, n_{k}}\right|\left(\left\|f_{2}^{(r)}\right\|_{\Delta_{k}} \|_{\infty}+K_{r}^{* *}\right)}{|I|^{2-r}-\left\|\Delta_{k}\right\|^{2-r} \max _{1 \leq n_{k} \leq N_{k}}\left|\alpha_{k, n_{k}}\right|} .
$$

From Ref. 21, pp. 28, classical cubic splines with boundary conditions of Type-I, Type-II or Type-III satisfy,

$$
\begin{gathered}
\left\|\Phi^{(r)}-S_{\Delta_{k}}^{(r)}\right\|_{\infty} \leq 5\left\|\Delta_{k}\right\|^{2-r} \omega\left(\Phi^{(r)} ;\left\|\Delta_{k}\right\|\right), \\
r=0,1,2,
\end{gathered}
$$

where $\omega(\Phi ; x)$ is the modulus of continuity of $\Phi(x)$. Inequality (3.13) gives

$$
\left\|S_{\Delta_{k}}^{(r)}\right\|_{\infty} \leq\left\|\Phi^{(r)}\right\|_{\infty}+5\left\|\Delta_{k}\right\|^{2-r} \omega\left(\Phi^{(r)} ;\left\|\Delta_{k}\right\|\right) .
$$

From inequality (3.9), with the same boundary conditions, $f_{2_{\Delta_{k}}}$ and $S_{\Delta_{k}}$ satisfy

$$
\begin{aligned}
\left\|f_{2}{ }_{\Delta_{k}}^{(r)}-S_{\Delta_{k}}^{(r)}\right\|_{\infty} \leq & \frac{\left\|\Delta_{k}\right\|^{2-r} s}{|I|^{2-r}-\left\|\Delta_{k}\right\|^{2-r} s} \\
& \times\left(\left\|S_{\Delta_{k}}^{(r)}\right\|_{\infty}+K_{r}\right) .
\end{aligned}
$$

Using inequality (3.14), $\left\|f_{2}{ }_{\Delta_{k}}^{(r)}\right\|_{\infty}$ exists as $k \rightarrow \infty$ for $r=0,1,2$. Inequalities (3.12) to (3.14) together with inequality (3.9) gives

$$
\left\|\Phi^{(r)}-f_{1}{ }_{\Delta_{k}}^{(r)}\right\|_{\infty} \leq\left\|\Delta_{k}\right\|^{2-r}\left\{5 \omega\left(\Phi^{(r)} ;\left\|\Delta_{k}\right\|\right)+\frac{\max _{1 \leq n_{k} \leq N_{k}}\left\{\left|\alpha_{k, n_{k}}\right|,\left|\beta_{k, n_{k}}\right|\right\}\left(\left\|S_{\Delta_{k}}^{(r)}\right\|_{\infty}+\left\|f_{2}{ }_{\Delta_{k}}^{(r)}\right\|_{\infty}+K_{r}^{*}+K_{r}^{* *}\right)}{|I|^{2-r}-\left\|\Delta_{k}\right\|^{2-r} \max _{1 \leq n_{k} \leq N_{k}}\left|\alpha_{k, n_{k}}\right|}\right\} .
$$

By the assumptions $\Phi \in C^{2}(I)$ and $\max _{1 \leq n_{k} \leq N_{k}}\left\{\left|\alpha_{k, n_{k}}\right|,\left|\beta_{k, n_{k}}\right|\right\} \leq\left\|\Delta_{k}\right\|<1$, the right hand side of inequality (3.15) tends to zero as $k \rightarrow \infty$. Hence, the convergence result (3.10) for the Class A follows from inequality (3.15). Since $\max _{1 \leq n_{k} \leq N_{k}}\left|\alpha_{n_{k}}\right| \leq s^{*}<1$ and $\max _{1 \leq n_{k} \leq N_{k}}\left|\beta_{n_{k}}\right|<s$, inequality (3.15) reduces to

$$
\left\|\Phi^{(r)}-f_{1 \Delta_{k}}^{(r)}\right\|_{\infty} \leq\left\|\Delta_{k}\right\|^{2-r}\left\{5 \omega\left(\Phi^{(r)} ;\left\|\Delta_{k}\right\|\right)+\frac{\max \left\{s^{*}, s\right\}\left(\left\|S_{\Delta_{k}}^{(r)}\right\|_{\infty}+\left\|f_{2}{ }^{(r)}\right\|_{k}+K_{r}^{*}+K_{r}^{* *}\right)}{|I|^{2-r}-\left\|\Delta_{k}\right\|^{2-r} s^{*}}\right\}
$$

Finally, the convergence result (3.11) for Class B follows from the above inequality.

The convergence results of cubic spline CHFIFs to the function $\Phi$ in $C^{3}\left[x_{0}, x_{N}\right]$ are given in the following if cubic spline fractal functions $f_{2 \Delta_{k}}$ satisfy any boundary conditions of Type-I, Type-II, or Type-III on a sequence of meshes.

Theorem 3.2. Let $\Phi \in C^{3}\left[x_{0}, x_{N}\right]$ and cubic spline CHFIFs $f_{1 \Delta_{k}}(x)$ satisfy boundary conditions of Type-I, Type-II or Type-III on a sequence of meshes $\left\{\Delta_{k}\right\}$ on $\left[x_{0}, x_{N}\right]$ with

$$
\lim _{k \rightarrow \infty}\left\|\Delta_{k}\right\|=0 \quad \text { and } \quad \frac{\left\|\Delta_{k}\right\|}{\min _{1 \leq n_{k} \leq N_{k}} h_{k, n_{k}}} \leq \theta<\infty
$$

If $\left\{\Delta_{k}\right\}$ is in Class $A$ or Class $B$, then respectively for $r=0,1,2,\left\|\Phi^{(r)}-f_{1}{ }_{\Delta_{k}}^{(r)}\right\|_{\infty}=\circ\left(\left\|\Delta_{k}\right\|^{2-r}\right)$ or $\left\|\Phi^{(r)}-f_{1 \Delta_{k}}^{(r)}\right\|_{\infty}=\bigcirc\left(\left\|\Delta_{k}\right\|^{2-r}\right)$.

Proof. It is known (Ref. 21, pp. 32) that classical cubic splines with boundary conditions of Type-I, Type-II or Type-III, satisfy

$$
\begin{gathered}
\left\|\Phi^{(r)}-S_{\Delta_{k}}^{(r)}\right\|_{\infty} \leq \frac{5}{3}\left\|\Delta_{k}\right\|^{3-r}(3+\bar{K}) \omega\left(\Phi^{(3)} ;\left\|\Delta_{k}\right\|\right), \\
r=0,1,2,
\end{gathered}
$$

where $\bar{K}=8 \theta^{2}(1+2 \theta)(1+3 \theta)$. From inequalities (3.9) and (3.16), it is clear that $\left\|S_{\Delta_{k}}^{(r)}\right\|_{\infty}$ and $\left\|f_{2}{ }_{\Delta_{k}}^{(r)}\right\|_{\infty}$ are bounded. Thus, the following error estimate holds for cubic spline CHFIFs with boundary conditions of Type-I, Type-II or Type-III:

$$
\begin{aligned}
& \left\|\Phi^{(r)}-f_{1 \Delta_{k}}^{(r)}\right\|_{\infty} \leq\left\|\Delta_{k}\right\|^{2-r}\left\{\frac{5}{3}\left\|\Delta_{k}\right\|(3+\bar{K}) \omega\left(\Phi^{(3)} ;\left\|\Delta_{k}\right\|\right)\right. \\
& \left.+\frac{\max _{1 \leq n_{k} \leq N_{k}}\left\{\left|\alpha_{k, n_{k}}\right|,\left|\beta_{k, n_{k}}\right|\right\}\left(\left\|S_{\Delta_{k}}^{(r)}\right\|_{\infty}+\left\|f_{2}^{(r)}\right\|_{\Delta_{k}} \|_{\infty}+K_{r}^{*}+K_{r}^{* *}\right)}{|I|^{2-r}-\left\|\Delta_{k}\right\|^{2-r} \max _{1 \leq n_{k} \leq N_{k}}\left|\alpha_{k, n_{k}}\right|}\right\} .
\end{aligned}
$$


The above estimate gives the convergence results of Theorem 3.2 depending on the sequence mesh of Class A or Class B.

The convergence results of cubic spline CHFIFs to the function $\Phi$ in $C^{4}\left[x_{0}, x_{N}\right]$ are given in the following if cubic spline fractal functions $f_{2 \Delta_{k}}$ satisfy any boundary conditions of Type-I or Type-II on a sequence of meshes.

Theorem 3.3. Let $\Phi \in C^{4}\left[x_{0}, x_{N}\right]$ and cubic spline CHFIFs $f_{1 \Delta_{k}}(x)$ satisfy boundary conditions of Type-I or Type-II on a sequence of meshes $\left\{\Delta_{k}\right\}$ on $\left[x_{0}, x_{N}\right]$ with $\lim _{k \rightarrow \infty}\left\|\Delta_{k}\right\|=0$ and $\frac{\left\|\Delta_{k}\right\|}{\min _{1 \leq n_{k} \leq N_{k}} h_{k, n_{k}}} \leq \eta<\infty$. If $\left\{\Delta_{k}\right\}$ is in Class
$A$ or Class $B$, then respectively for $r=0,1,2$, $\left\|\Phi^{(r)}-f_{1 \Delta_{k}}^{(r)}\right\|_{\infty}=\circ\left(\left\|\Delta_{k}\right\|^{2-r}\right)$ or $\left\|\Phi^{(r)}-f_{1 \Delta_{k}}^{(r)}\right\|_{\infty}=$ $\bigcirc\left(\left\|\Delta_{k}\right\|^{2-r}\right)$.

Proof. It is known ${ }^{22}$ that, classical cubic splines with boundary conditions of Type-I or Type-II, satisfy

$$
\begin{array}{r}
\left\|\Phi^{(r)}-S_{\Delta_{k}}^{(r)}\right\|_{\infty} \leq L_{r}\left\|\Phi^{(4)}\right\|_{\infty}\left\|\Delta_{k}\right\|^{4-r}, \\
r=0,1,2,3,
\end{array}
$$

where $L_{0}=5 / 384, L_{1}=1 / 24, L_{2}=3 / 8$, and $L_{3}=\left(\eta+\eta^{-1}\right) / 2$. From inequalities (3.9) and (3.17) it follows that $\left\|S_{\Delta_{k}}^{(r)}\right\|_{\infty}$ and $\left\|f_{2}{ }_{\Delta_{k}}^{(r)}\right\|_{\infty}$ are bounded. Hence, the error estimate in this case for cubic spline CHFIFs with boundary conditions of Type-I or Type-II, is given by

$$
\begin{aligned}
& \left\|\Phi^{(r)}-f_{1}{ }_{\Delta_{k}}^{(r)}\right\|_{\infty} \leq\left\|\Delta_{k}\right\|^{2-r}\left\{L_{r}\left\|\Phi^{(4)}\right\|_{\infty}\left\|\Delta_{k}\right\|^{2}\right. \\
& \left.+\frac{\max _{1 \leq n_{k} \leq N_{k}}\left\{\left|\alpha_{k, n_{k}}\right|,\left|\beta_{k, n_{k}}\right|\right\}\left(\left\|S_{\Delta_{k}}^{(r)}\right\|_{\infty}+\left\|f_{2}{ }_{\Delta_{k}}^{(r)}\right\|_{\infty}+K_{r}^{*}+K_{r}^{* *}\right)}{|I|^{2-r}-\left\|\Delta_{k}\right\|^{2-r} \max _{1 \leq n_{k} \leq N_{k}}\left|\alpha_{k, n_{k}}\right|}\right\}, \quad r=0,1,2 .
\end{aligned}
$$

Thus, the above inequality gives the convergence results of Theorem 3.3.

Remark 3.2. The spline CHFIF $f_{1}$ uniformly converge in the $C^{1}$ norm to the data generating function $\Phi$ if $\left\{\Delta_{k}\right\}$ is in Class B. If there exists a positive number $u$ such that $\max _{1 \leq n_{k} \leq N_{k}}\left\{\left|\alpha_{k, n_{k}}\right|,\left|\beta_{k, n_{k}}\right|\right\} \leq$ $\left\|\Delta_{k}\right\|^{u}$ for all $k=0,1,2, \ldots$, then $f_{1}$ converge uniformly to $\Phi$ in the $C^{2}$ norm on $I$.

\section{EXAMPLES OF CUBIC SPLINE CHFIFs}

In this section, we construct examples of cubic spline CHFIFs as the fixed point of the IFS given by Eq. (2.8). Suppose that $\left\{(0,0),\left(\frac{2}{5}, 1\right)\right.$, $\left.\left(\frac{3}{4},-1\right),(1,2)\right\}$ is the given interpolation data for cubic spline CHFIFs. Chose free variables $\alpha_{n}=0.8$, $n=1,2,3$; hidden variables $z_{0}=3, z_{1}=2, z_{2}=$ $8, z_{3}=5, \gamma_{1}=0.3, \gamma_{2}=0.35, \gamma_{3}=0.4$; and constrained free variables $\beta_{1}=0.4, \beta_{2}=0.6, \beta_{3}=$ 0.5. Using Eq. (2.1), each IFS has $L_{1}(x)=\frac{2}{5} x$, $L_{2}(x)=\frac{7}{20} x+\frac{2}{5}$, and $L_{3}(x)=\frac{1}{4} x+\frac{3}{4}$. For the first two examples, we compute $F_{n}^{2}(x, z)$ for the self-affine cubic spline fractal function $f_{2}$ with a boundary condition $f_{2}{ }^{\prime}\left(x_{0}\right)=10$ and $f_{2}{ }^{\prime}\left(x_{3}\right)=1$. The moments are evaluated (Table 1) by using the system of equations (see Appendix). These moments are used in Eq. (2.10) for the construction of $F_{n}^{2}(x, z)$ (Table 2). For constructing an example of the cubic spline CHFIF with boundary conditions of Type-I, we choose $f_{1}{ }^{\prime}\left(x_{0}\right)=2$ and $f_{1}{ }^{\prime}\left(x_{3}\right)=5$. Equations (A.3) are solved with these choices to get

Table 1 Derivatives Used for IFSs of CHFIFs.

\begin{tabular}{ccccccccccccc}
\hline Fig. & $\boldsymbol{f}_{\mathbf{2}}{ }^{\prime}\left(\boldsymbol{x}_{\mathbf{0}}\right)$ & $\boldsymbol{M}_{\mathbf{0}}$ & $\boldsymbol{M}_{\mathbf{1}}$ & $\boldsymbol{M}_{\mathbf{2}}$ & $\boldsymbol{M}_{\mathbf{3}}$ & $\boldsymbol{f}_{\mathbf{2}}{ }^{\prime}\left(\boldsymbol{x}_{\mathbf{3}}\right)$ & $\boldsymbol{f}_{\mathbf{1}}{ }^{\prime}\left(\boldsymbol{x}_{\mathbf{0}}\right)$ & $\boldsymbol{M}_{\mathbf{0}}^{*}$ & $\boldsymbol{M}_{\mathbf{1}}^{*}$ & $\boldsymbol{M}_{\mathbf{2}}^{*}$ & $\boldsymbol{M}_{\mathbf{3}}^{*}$ & $\boldsymbol{f}_{\mathbf{1}}{ }^{\prime}\left(\boldsymbol{x}_{\mathbf{3}}\right)$ \\
\hline 1 & 10 & -209.49 & 236.76 & -235.69 & 388.58 & 1 & 2 & -0.66 & -90.65 & 156.63 & -131.82 & 5 \\
2 & 10 & -209.49 & 236.76 & -235.69 & 388.58 & 1 & 2 & 201.38 & -123.87 & 113.56 & -50.32 & 5 \\
3 & 10 & -418.8 & 923.6 & -836.1 & 1933.1 & 1 & 2 & 921.8 & 1420.3 & 1614.3 & 1646.2 & 5 \\
4 & -13.43 & 10 & 122.47 & -171.90 & 1 & -21.64 & 2 & -95.98 & -289.22 & -29.01 & -383.10 & 5 \\
5 & 10 & -146.01 & 125.51 & -197.81 & 202.07 & 1 & 2 & -67.79 & -235.24 & 21.97 & -316.34 & 5 \\
6 & 2 & -77.87 & -331.38 & -59.68 & -462.54 & 5 & 2 & -77.87 & -331.38 & -59.68 & -462.54 & 5 \\
\hline
\end{tabular}




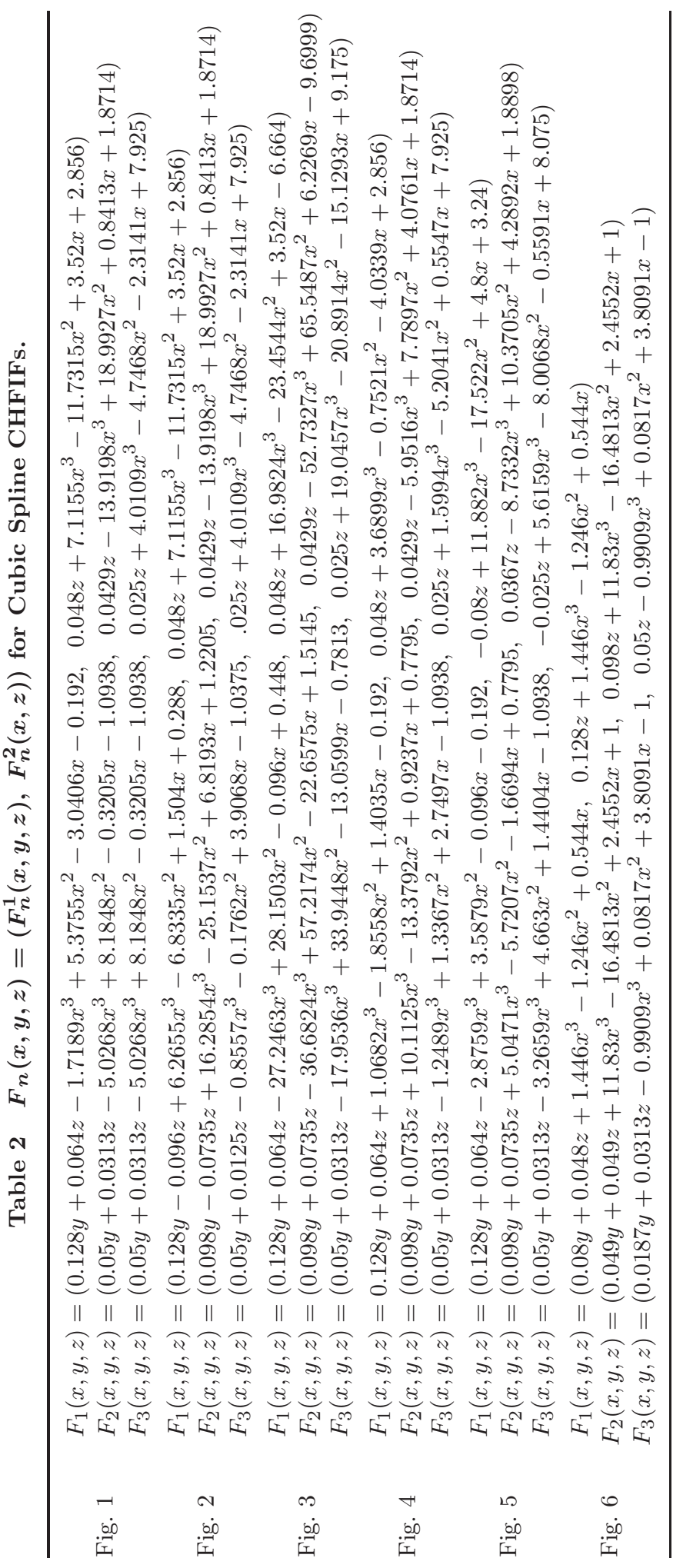


the values of moments $M_{0}^{*}, M_{1}^{*}, M_{2}^{*}, M_{3}^{*}$ (Table 1 ). These moments are now used in Eq. (2.9) for the construction of $F_{n}^{1}(x, y, z)$ (Table 2). Iterations of the IFS code (2.8) generates the desired cubic spline CHFIF (Fig. 1). To illustrate the effect of constrained free variables on the shape of the cubic spline CHFIF in comparison with Fig. 1, we take $\beta_{1}=\beta_{2}=-0.6$, and $\beta_{3}=0.2$. Using the computed values of moments $M_{0}^{*}, M_{1}^{*}, M_{2}^{*}, M_{3}^{*}$ (Table 1 ), we evaluate $F_{n}^{1}(x, y, z), n=1,2,3$ (Table 2 ). Iterations of the IFS code (2.8) generates the desired cubic spline CHFIF (Fig. 2). Similarly, perturbations in the free variables $\alpha_{n}$ would affect the shape of the cubic spline CHFIF.

The effect of change in hidden variables, i.e. parameter $z_{n}$, boundary conditions of fractal function $f_{2}$ and free variables $\gamma_{n}$, on the shape of the cubic spline CHFIF are illustrated in Figs. 3, 4 and 5 , respectively by comparing these with Fig. 1 . In Fig. 3, we only modify free parameters as $z_{0}=-7$, $z_{1}=-10, z_{2}=9, z_{3}=-8$. In Fig. 4, we choose boundary conditions for self-affine fractal function $f_{2}$ as $M_{0}=10, M_{3}=1$ instead of $f_{2}^{\prime}\left(x_{0}\right)=10$,

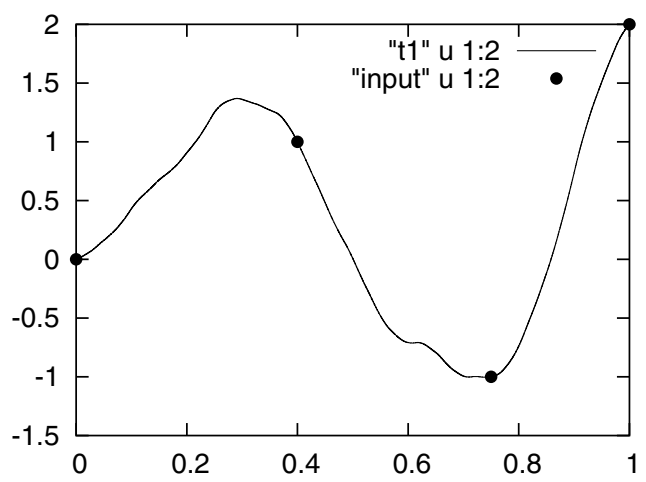

Fig. 1 Cubic spline CHFIF with boundary conditions of Type-I.

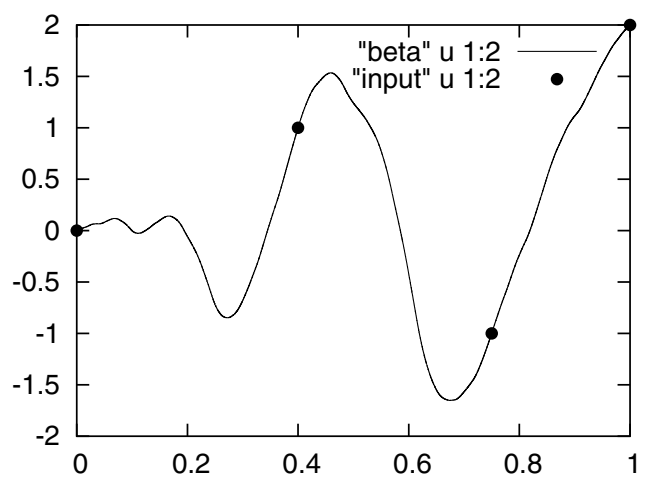

Fig. 2 Cubic spline CHFIF with boundary conditions of Type-I and different $\beta_{n}$.

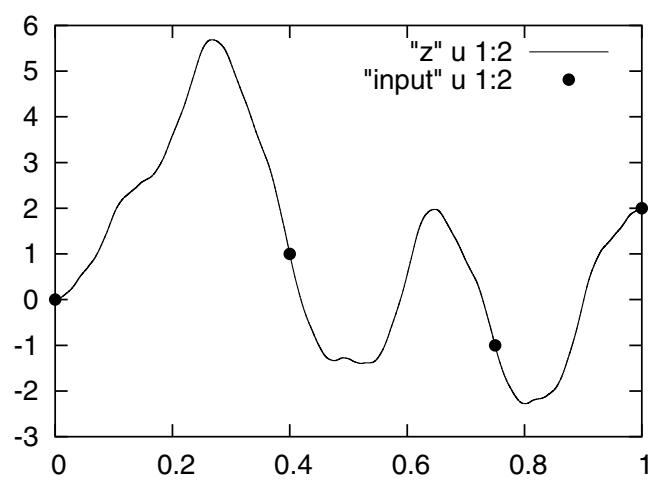

Fig. 3 Cubic spline CHFIF with boundary conditions of Type-I and different $z_{n}$.

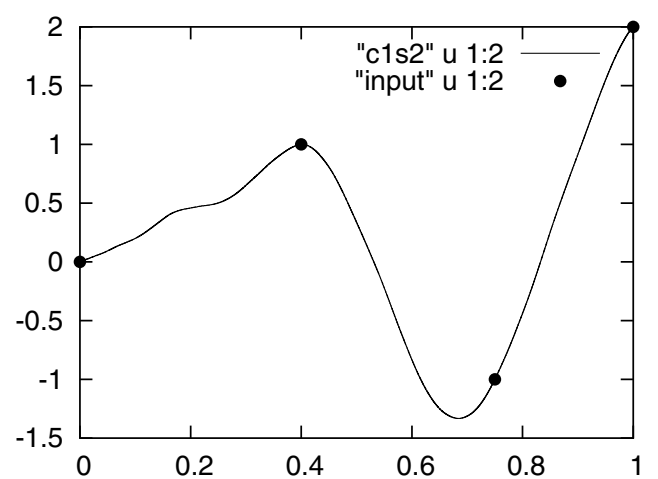

Fig. 4 Cubic spline CHFIF with boundary conditions of Type-I and different boundary conditions for self-affine cubic spline fractal function.

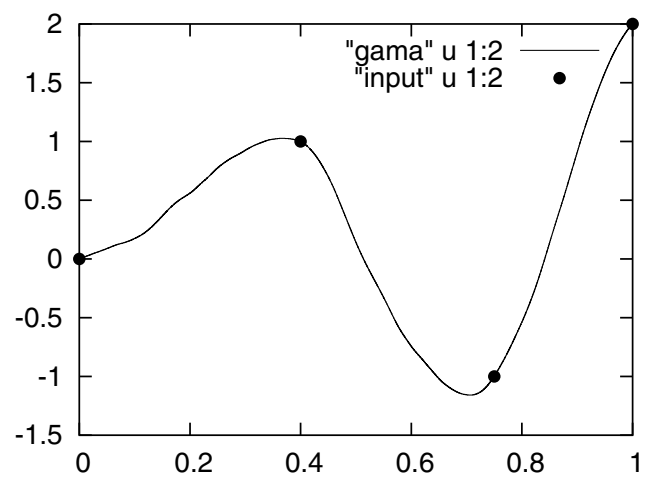

Fig. 5 Cubic spline CHFIF with boundary conditions of Type-I and different $\gamma_{n}$.

$f_{2}^{\prime}\left(x_{3}\right)=1$. In Fig. 5, we change only free variables $\gamma_{1}=-0.5, \quad \gamma_{2}=0.3$ and $\gamma_{3}=-0.4$. Depending on changes in hidden variables, moments of the self-affine cubic spline fractal function and cubic spline CHFIF are calculated (Table 1). These are used to determine $F_{n}(x, y, z)$ (Table 2) for the IFS code (2.8). Finally, we assume $y_{n}=z_{n}$ for $n=0,1,2,3, \alpha_{n}+\beta_{n}=\gamma_{n}$, i.e. $\alpha_{1}=$ $\beta_{3}=0.5, \alpha_{2}=\beta_{2}=0.4$ and $\alpha_{3}=\beta_{1}=0.5$ 


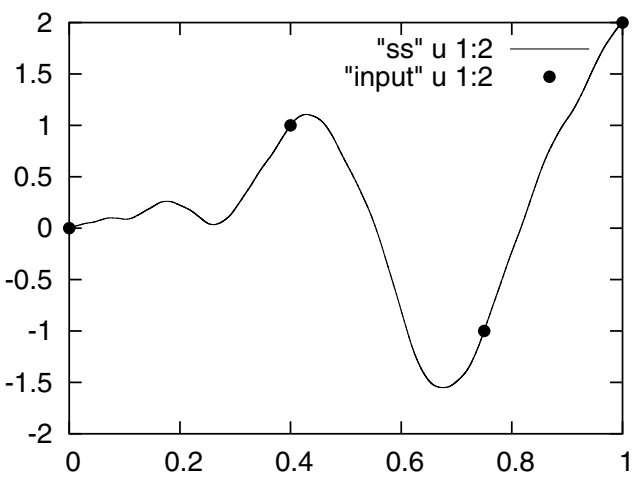

Fig. 6 Self-affine cubic spline CHFIF.

with the same boundary conditions. In this case, the projection of the attractor generates the self-affine cubic spline CHFIF (Fig. 6). Hence, our approach offers more flexibility and diversity in the choice of self-affine or non-self-affine cubic spline CHFIFs to an experimenter depending on the need of the problem.

\section{CONCLUSION}

The construction of the cubic spline CHFIF through moments is initiated for the first time to approximate non-self-affine smooth objects. This construction allows admissibility of any kind of boundary conditions and generalizes results of the classical cubic spline.

For a data generating function $\Phi \in C^{r}\left[x_{0}, x_{n}\right]$, $r=2,3$, or 4 , it is proved that, cubic spline CHFIFs converge to $\Phi$ with arbitrary degree of accuracy when the step size approaches zero on two different classes of mesh. These upper bounds on error in approximation of $\Phi$ and its derivatives by the cubic spline CHFIF and its derivatives respectively with different boundary conditions are also obtained. If the data generating function $\Phi(x)$ satisfies $\omega\left(\Phi^{(2)}, t\right)=\bigcirc\left(|t|^{\mu}(\log |t|)^{n}\right), n=0,1$, or 2 , $0<\mu<1, \Phi$ can be approximated satisfactorily by a cubic spline CHFIF $f_{1}(x)$ by choosing only free variables $\alpha_{n}$ and $\gamma_{n}$ suitably, since $\beta_{n}$ and $z_{n}$ do not affect the smoothness of the CHFIF $f_{1}^{\prime \prime}(x) .{ }^{14}$

Hidden variables, free variables and constrained variables play an important role in determining the shape of the cubic spline CHFIF. For prescribed boundary conditions, an infinite number of cubic spline CHFIFs can be constructed interpolating the same data by changing free variables $\alpha_{n}$, constrained free variables $\beta_{n}$, hidden variables $\gamma_{n}$, free parameter $z_{n}$ or boundary conditions of self-affine cubic spline fractal function. Thus, for simulating objects having non-self-affine or self-affine smooth shapes, the cubic spline CHFIF offers more flexibility. It is felt that spline FIF should find rich applications since classical splines have vast applications in $\mathrm{CAM} / \mathrm{CAD}$ and other mathematical, engineering applications. ${ }^{19,20}$ The self-affine and non-selfaffine nature of smooth objects in various scientific applications can also be effectively captured with the use of cubic spline CHFIFs.

\section{ACKNOWLEDGMENTS}

The authors are thankful to the referee for his/her valuable suggestions. The work was partially supported by the CSIR, India, Grant No.: 9/92(160)/ 98-EMR-I.

\section{REFERENCES}

1. B. B. Mandelbrot, The Fractal Geometry of Nature (W. H. Freeman, San Francisco, 1983).

2. B. B. Mandelbrot, Fractals and Scaling in Finance (Springer-Verlag, New York, 1997).

3. J. Feder, Fractals (Plenum Press, New York 1988).

4. M. Frame and B. B. Mandelbrot, Fractals, Graphics, and Mathematics Education (The Mathematical Association of America, Washington, 2000).

5. B. J. West, Physiology, Promiscuity and Prophecy at the Millennium: A Tale of Tails, in Studies of Nonlinear Phenomena in the Life Sciences, Vol. 9 (World Scientific, Singapore, 1998).

6. J. L. Véhel, K. Daoudi and E. Lutton, Fractal modelling of speech signals, Fractals 2(3) (1994) 379-382.

7. Y. Fisher, Fractal Image Compression (SpringerVerlag, New York, 1996).

8. M. F. Barnsley, Fractal functions and interpolations, Constr. Approx. 2 (1986) 303-329.

9. M. F. Barnsley, Fractals Everywhere (Academic Press, Orlando, Florida, 1988).

10. M. F. Barnsley, J. Elton, D. P. Hardin and P. R. Massopust, Hidden variable fractal interpolation functions, SIAM J. Math. Anal. 20(5) (1989) 1218-1242.

11. P. R. Massopust, Fractal functions, in Fractal Surfaces and Wavelets (Academic Press, 1994).

12. J. Santamaria, M. E. Gómez, J. L. Vicent, K. M. Krishnan and I. K. Schuller, Scaling of the interface roughness in Fe-Cr superlattices: self-affine versus non-self-affine, Phys. Rev. Lett. 19 (2002) 190601.

13. A. K. B. Chand and G. P. Kapoor, Hidden variable bivariate fractal interpolation surfaces, Fractals 11(3) (2003) 277-288.

14. A. K. B. Chand, A study on coalescence and spline fractal interpolation functions, PhD thesis, IIT Kanpur, India (2005). 
15. M. F. Barnsley and A. N. Harrington, The calculus of fractal interpolation functions, J. Approx. Theory 57 (1989) 14-34.

16. A. K. B. Chand and G. P. Kapoor, Generalized cubic spline fractal interpolation functions, SIAM J. Numer. Anal. 44(2) (2006) 655-676.

17. M. A. Navascués and M. V. Sebastián, Some results on convergence of cubic spline fractal interpolation functions, Fractals 11(1) (2003) 1-7.

18. M. A. Navascués and M. V. Sebastián, Smooth fractal interpolation, J. Inequalities Appl., Article ID 78734 (2006) 1-20.

19. G. Farin, Curves and Surfaces for Computer Aided Geometric Design: A Practical Guide (Academic Press, San Diego, 1990).

20. G. D. Knott, Interpolating Cubic Splines (Birkhäuser, Boston, 2000).

21. J. Ahlberg, E. Nilson and J. Walsh, The Theory of Splines and Their Applications (Academic Press, 1967).

22. C. A. Hall and W. W. Meyer, Optimal error bounds for cubic spline interpolation, J. Approx. Theory $\mathbf{1 6}$ (1976) 105-122.

\section{APPENDIX}

In this section, the details of employing moments $M_{n}^{*}$ and $M_{n} ; n=0,1,2, \ldots, N$ in the construction of cubic spline CHFIFs in Sec. 2 are given. Since $f_{1}^{\prime \prime}$ is affine,

$$
\begin{aligned}
& f_{1}^{\prime \prime}\left(L_{n}(x)\right) \\
& =\alpha_{n} f_{1}^{\prime \prime}(x)+\beta_{n} f_{2}^{\prime \prime}(x)+\frac{k_{n}\left(x-x_{0}\right)}{x_{N}-x_{0}}+l_{n}, \\
& n=1,2, \ldots, N . \quad \text { A. } 1)
\end{aligned}
$$

Using Eq. (2.1) and Eq. (A.1), $k_{n}=M_{n}^{*}-M_{n-1}^{*}-$ $\alpha_{n}\left(M_{N}^{*}-M_{0}^{*}\right)-\beta_{n}\left(M_{N}-M_{0}\right)$ and $l_{n}=M_{n-1}^{*}-$ $\alpha_{n} M_{0}^{*}-\beta_{n} M_{0}$. So, Eq. (A.1) reduces to

$$
\begin{aligned}
f_{1}^{\prime \prime}\left(L_{n}(x)\right)= & \alpha_{n} f_{1}^{\prime \prime}(x)+\beta_{n} f_{2}^{\prime \prime}(x) \\
& +\frac{\left(M_{n}^{*}-\alpha_{n} M_{N}^{*}-\beta_{n} M_{N}\right)\left(x-x_{0}\right)}{x_{N}-x_{0}} \\
& +\frac{\left(M_{n-1}^{*}-\alpha_{n} M_{0}^{*}-\beta_{n} M_{0}\right)\left(x_{N}-x\right)}{x_{N}-x_{0}} .
\end{aligned}
$$

Integrating the above equations twice and using Eq. (2.1), the cubic spline CHFIF satisfies

$$
\begin{aligned}
f_{1}\left(L_{n}(x)\right)= & a_{n}^{2}\left\{\alpha_{n} f_{1}(x)+\beta_{n} f_{2}(x)+\frac{\left(M_{n}^{*}-\alpha_{n} M_{N}^{*}-\beta_{n} M_{N}\right)\left(x-x_{0}\right)^{3}}{6\left(x_{N}-x_{0}\right)}\right. \\
& +\frac{\left(M_{n-1}^{*}-\alpha_{n} M_{0}^{*}-\beta_{n} M_{0}\right)\left(x_{N}-x\right)^{3}}{6\left(x_{N}-x_{0}\right)}-\frac{\left(M_{n-1}^{*}-\alpha_{n} M_{0}^{*}-\beta_{n} M_{0}\right)\left(x_{N}-x_{0}\right)\left(x_{N}-x\right)}{6} \\
& -\frac{\left(M_{n}^{*}-\alpha_{n} M_{N}^{*}-\beta_{n} M_{N}\right)\left(x_{N}-x_{0}\right)\left(x-x_{0}\right)}{6}+\left(\frac{y_{n-1}}{a_{n}^{2}}-\alpha_{n} y_{0}-\beta_{n} z_{0}\right) \frac{x_{N}-x}{x_{N}-x_{0}} \\
& \left.+\left(\frac{y_{n}}{a_{n}^{2}}-\alpha_{n} y_{N}-\beta_{n} z_{N}\right) \frac{x-x_{0}}{x_{N}-x_{0}}\right\}, \quad n=1,2, \ldots, N .
\end{aligned}
$$

Introduce the following notations:

$$
\begin{aligned}
h_{n}= & x_{n}-x_{n-1}, \quad C_{n}^{*}=\frac{-6 a_{n+1} \alpha_{n+1}}{h_{n}+h_{n+1}}, \quad C_{n}=\frac{-\left(\alpha_{n} h_{n}+2 \alpha_{n+1} h_{n+1}\right)}{h_{n}+h_{n+1}}, \quad \lambda_{n}=\frac{h_{n+1}}{h_{n}+h_{n+1}}, \\
d_{n}^{*}= & \frac{6\left[\left(y_{n+1}-y_{n}\right) / h_{n+1}-\left(y_{n}-y_{n-1}\right) / h_{n}\right]}{h_{n}+h_{n+1}}-\frac{6\left(a_{n+1} \alpha_{n+1}-a_{n} \alpha_{n}\right)}{h_{n}+h_{n+1}} \frac{y_{N}-y_{0}}{x_{N}-x_{0}} \\
& -\frac{6\left(a_{n+1} \beta_{n+1}-a_{n} \beta_{n}\right)}{h_{n}+h_{n+1}} \frac{z_{N}-z_{0}}{x_{N}-x_{0}}+\frac{6\left(a_{n+1} \beta_{n+1} f_{2}^{\prime}\left(x_{0}\right)-a_{n} \beta_{n} f_{2}^{\prime}\left(x_{N}\right)\right)}{h_{n}+h_{n+1}} \\
& +\frac{\beta_{n} h_{n}+2 \beta_{n+1} h_{n+1}}{h_{n}+h_{n+1}} M_{0}+\frac{2 \beta_{n} h_{n}+\beta_{n+1} h_{n+1}}{h_{n}+h_{n+1}} M_{N} \\
\mu_{n}= & 1-\lambda_{n}, \quad D_{n}=\frac{-\left(2 \alpha_{n} h_{n}+\alpha_{n+1} h_{n+1}\right)}{h_{n}+h_{n+1}}, \quad D_{n}^{*}=\frac{6 a_{n} \alpha_{n}}{h_{n}+h_{n+1}}, \quad n=1,2, \ldots, N-1, \\
d_{0}^{*}= & 6 / h_{1}\left[y_{1}-y_{0}-\alpha_{1} a_{1}^{2}\left(y_{N}-y_{0}\right)-\beta_{1} a_{1}^{2}\left(z_{N}-z_{0}\right)\right]+\beta_{1}\left[6 a_{1} f_{2}{ }^{\prime}\left(x_{0}\right)+2 h_{1} M_{0}+h_{1} M_{N}\right],
\end{aligned}
$$




$$
\begin{aligned}
C_{0}^{*}= & 6\left(1-a_{1} \alpha_{1}\right), \quad C_{0}=2\left(1-\alpha_{1}\right) h_{1}, \quad \lambda_{0}=h_{1}, \quad D_{0}=-\alpha_{1} h_{1}, \quad C_{N}=-\alpha_{N} h_{N}, \quad \mu_{N}=h_{N}, \\
D_{N}= & 2\left(1-\alpha_{N}\right) h_{N}, \quad D_{N}^{*}=-6\left(1-a_{N} \alpha_{N}\right), \quad d_{N}^{*}=-6 / h_{N}\left[y_{N}-y_{N-1}-\alpha_{N} a_{N}^{2}\left(y_{N}-y_{0}\right)\right. \\
& \left.-\beta_{N} a_{N}^{2}\left(z_{N}-z_{0}\right)\right]+\beta_{N}\left[h_{N} M_{0}+2 h_{N} M_{N}-6 a_{N} f_{2}{ }^{\prime}\left(x_{N}\right)\right] .
\end{aligned}
$$

Using Eq. (A.2), we can write the functional relation for $f_{1}^{\prime}\left(x_{0}\right)$, continuity relations at $f_{1}^{\prime}\left(x_{n}\right)$ for $n=$ $1,2, \cdots, N-1$, and the functional relation for $f_{1}^{\prime}\left(x_{N}\right)$ in the matrix form as

$$
\left[\begin{array}{ccccccccccc}
C_{0}^{*} & C_{0} & \lambda_{0} & 0 & 0 & \ldots & 0 & 0 & 0 & D_{0} & 0 \\
C_{1}^{*} & C_{1}+\mu_{1} & 2 & \lambda_{1} & 0 & \ldots & 0 & 0 & 0 & D_{1} & D_{1}^{*} \\
C_{2}^{*} & C_{2} & \mu_{2} & 2 & \lambda_{2} & \ldots & 0 & 0 & 0 & D_{2} & D_{2}^{*} \\
C_{3}^{*} & C_{3} & 0 & \mu_{3} & 2 & \ldots & 0 & 0 & 0 & D_{3} & D_{3}^{*} \\
\vdots & \vdots & \vdots & \vdots & \vdots & & \vdots & \vdots & \vdots & \vdots & \vdots \\
C_{N-3}^{*} & C_{N-3} & 0 & 0 & 0 & \ldots & 2 & \lambda_{N-3} & 0 & D_{N-3} & D_{N-3}^{*} \\
C_{N-2}^{*} & C_{N-2} & 0 & 0 & 0 & \ldots & \mu_{N-2} & 2 & \lambda_{N-2} & D_{N-2} & D_{N-2}^{*} \\
C_{N-1}^{*} & C_{N-1} & 0 & 0 & 0 & \ldots & 0 & \mu_{N-1} & 2 & \lambda_{N-1}+D_{N-1} & D_{N-1}^{*} \\
0 & C_{N} & 0 & 0 & 0 & \ldots & 0 & 0 & \mu_{N} & D_{N} & D_{N}^{*}
\end{array}\right]\left[\begin{array}{c}
f_{1}{ }^{\prime}\left(x_{0}\right) \\
M_{0}^{*} \\
M_{1}^{*} \\
M_{2}^{*} \\
\vdots \\
M_{N-2}^{*} \\
M_{N-1}^{*} \\
M_{N}^{*} \\
f_{1}{ }^{\prime}\left(x_{N}\right)
\end{array}\right]=\left[\begin{array}{c}
d_{0}^{*} \\
d_{1}^{*} \\
d_{2}^{*} \\
d_{3}^{*} \\
\vdots \\
d_{N-3}^{*} \\
d_{N-2}^{*} \\
d_{N-1}^{*} \\
d_{N}^{*}
\end{array}\right] .
$$

The system of Eqs. (A.3) consisting of $(N+1) \times(N+$ 3) coefficient matrix has unknowns $f_{1}{ }^{\prime}\left(x_{0}\right), M_{0}^{*}$, $M_{1}^{*}, \ldots, M_{N}^{*}, f_{1}{ }^{\prime}\left(x_{N}\right)$.

First an analogue of Eq. (A.3), can be constructed for the self-affine fractal function $f_{2}$ by taking $\beta_{n}=0$ and $\alpha_{n}=\gamma_{n}$. The solution of the corresponding system of equations with suitable boundary conditions determines $f_{2}{ }^{\prime}\left(x_{0}\right)$ and $f_{2}{ }^{\prime}\left(x_{N}\right)$ and moments $M_{n}, n=0,1,2, \ldots, N$ of $f_{2}$.
Next, using values of $f_{2}{ }^{\prime}\left(x_{0}\right), f_{2}{ }^{\prime}\left(x_{N}\right), M_{0}$, and $M_{N}$, with suitable boundary conditions, the system of Eqs. (A.3) is solved and $f_{1}{ }^{\prime}\left(x_{0}\right), M_{0}^{*}, M_{1}^{*}, \ldots, M_{N}^{*}$, and $f_{1}{ }^{\prime}\left(x_{N}\right)$ are determined. These values of $M_{n}$ and $M_{n}^{*} ; n=0,1,2, \ldots, N$ are finally used in the construction of a cubic spline CHFIF from the IFS that is given by Eq. (2.8). 